Revue d'histoire de l'enfance « irrégulière »

Le Temps de l'histoire

$20 \mid 2018$

Sexualités juvéniles

Pratiques de négociations sexuelles des jeunes femmes pauvres dans l'Argentine des années 1940

Negociating Intimacies. Poor Women's Domestic Experiences in Mid-Twentieth

Century Buenos Aires

Patricio Simonetto

(2) OpenEdition

Journals

Édition électronique

URL : http://journals.openedition.org/rhei/4285

DOI : 10.4000/rhei.4285

ISSN : $1777-540 \mathrm{X}$

Éditeur

Presses universitaires de Rennes

Édition imprimée

Date de publication : 15 novembre 2018

Pagination : 85-104

ISBN : 978-2-7535-7571-4

ISSN : $1287-2431$

Référence électronique

Patricio Simonetto, «Pratiques de négociations sexuelles des jeunes femmes pauvres dans

l'Argentine des années 1940 », Revue d'histoire de l'enfance « irrégulière» [En ligne], 20 | 2018, mis en ligne le 15 novembre 2020, consulté le 03 décembre 2020. URL : http://journals.openedition.org/rhei/ 4285 ; DOI : https://doi.org/10.4000/rhei.4285 


\section{Pratiques de négociations sexuelles des jeunes femmes pauvres dans l'Argentine des années 1940}

À partir de trois situations de jeunes filles mineures l'article analyse les expériences domestiques féminines confrontées aux interventions publiques et privées dans le Sud de Buenos Aires au milieu du $x^{e}{ }^{e}$ siècle. Pour cela, est examiné comment les jeunes filles pauvres ont tenté de trouver une marge d'autonomie (économique et sexuelle) tout en négociant avec l'État, la famille et le couple. Dans une perspective intersectionnelle et historique, est interrogée l'utilité des concepts de genre, de classe et d'âge pour comprendre certaines expériences d'adolescentes prolétaires, nées à la périphérie de Buenos Aires. Ce travail s'appuie sur l'analyse d'archives judiciaires en matière de proxénétisme, viol, abandon du foyer, prostitution, et corruption des mineurs au Département Historique et Judiciaire de Dolores (DHJD) afin de reconstruire les histoires de vie de jeunes femmes pauvres.

Analyzing the life of three girls, the article aims to demonstrate how young women's domestic experiences are confronted with public and private interventions in South Buenos Aires in mid-twentieth century. The article examines how poor girls experienced their intimacy and domesticity combined with the negotiation with the State, families, and couples. In an intersectional and historical perspective, age, class and gender are three useful concepts to understand some experiences of life of poor women born in Buenos Aires rural areas. This research is based on the analysis of judicial records on pimping, rape, house abandonment, prostitution and minor corruption of the Departamento Histórico Judicial de Dolores (DHJD).

Mots-clés : domestiques, sexualité, intimité, justice, mineures, État, Argentine, Buenos Aires.

Keywords: domesticity, sexuality, intimacy, justice, minor girl, State, Argentina, Buenos Aires.

$\mathrm{E}$ n 1942, à Balcarce, une communauté rurale du Sud de la province de Buenos Aires (Argentine), María dénonce au commissariat le détournement de sa fille Elba, âgée de 15 ans. Elle déclare qu'elle vit avec un homme qui

\author{
Patricio Simonetto \\ Doctorant en histoire. \\ Centre d'études en \\ histoire, culture et \\ mémoire (CEHCMe), \\ Université nationale \\ de Quilmes, Argentine \\ Je remercie \\ la Dra. Maria Bjerg, \\ la Dra. Pamela \\ Fuentes Peralta, \\ la Dra. Carolina \\ Biernat et \\ la Dra. Andrea \\ Andújar pour leurs \\ commentaires. \\ J'exprime ma grati- \\ tude aux évaluateurs \\ de la RHEI pour leurs \\ remarquables apports \\ à mon article.
}


1. Département historique judiciaire Sur (DHJS), Dossier 508 : 22, 1942.

2. Allemandi Cecilia, Sirvientes, criadas y nodrizas. Una historia del servicio doméstico en la ciudad de Buenos Aires, Buenos Aires, Teseo, 2017; GuY Donna, Las mujeres y la construcción del Estado del Bienestar: caridad y creación de derechos en Argentina, Buenos Aires, Prometeo, 2011; StaGno

Leando, Una infancia aparte: la minoridad en la provincia de Buenos Aires (1930-1943), Buenos Aires, Flacso, 2010.

3. Le terme « rancho " est un concept autochtone utilisé pour nommer une construction particulière de la zone rurale de La Pampa aux murs en torchis et au toit de paille.
4. DHJS, Dossier $508: 22$,

1942.

5. Nous avons décidé d'utiliser le concept de mineur comme une catégorie initiale assignée par les médecins et juristes pour définir des enfants et des jeunes de moins de 21 ans avec des schémas de comportement, d'éducation, de travail et de sexualité ne faisant pas partie de ce qui est acceptable. De ce fait, cette condition réaffirme une

position sociale subalterne face aux adultes de leur famille, voisins et couples. Voir : ZAPIOLA María, « Ley de Patronato de Menores de 1919 : ¿una bisagra histórica? ", in LIONETTI

Lucía et Miguez Daniel, Las Infancias en la Historia

Argentina. Intersecciones entre Prácticas, Discursos

e Instituciones (1890-1960),

Buenos Aires, Prohistoria,

2010. la force à avoir des relations sexuelles avec d'autres hommes moyennant paiement. Elba a quitté sa famille quelques jours plus tôt, après avoir été placée par ses parents comme domestique chez des voisins aux revenus ${ }^{1}$ plus élevés. C'est alors une pratique très répandue dans les familles de travailleurs pauvres qui placent leurs enfants dans d'autres foyers ou au sein d'institutions soutenues par des organismes d'État ou de charité2.

Giménez, un boulanger espagnol de 39 ans, l'emploie comme servante moyennant le gîte et le couvert, ainsi qu'une petite rémunération. Pendant huit jours, elle fait le ménage et travaille dans la boutique et dans la maison du boulanger jusqu'à ce qu'elle abandonne son travail pour déménager dans un rancho $o^{3}$ avec Salvio, un journalier agricole et guitariste de quatorze ans son aîné, et deux autres hommes. Ils vivent tous ensemble dans un espace composé d'une cuisine et d'une chambre. Une fois la procédure judiciaire achevée, le juge décide de placer la jeune fille dans une institution pour mineures à La Plata, capitale de la province, jusqu'à sa majorité (21 ans), la séparant ainsi de ses parents, qu'il juge incompétents. Après un placement de six ans, Elba quitte l'institution sous la tutelle d'un adulte qui l'assiste pour trouver de nouveau un travail comme employée de maison ${ }^{4}$.

Cet article a pour objectif d'étudier les expériences sexuelles des jeunes filles pauvres prises en charge par la justice pour avoir proposé contre paiement des services sexuels ${ }^{5}$. Nous cherchons à comprendre comment et dans quelles conditions ces jeunes filles négocient leur "intimité » avec l'État, incarné par le tribunal correctionnel et criminel, mais aussi avec leur famille et leur 
couple, en essayant de parvenir à une autonomie relative, par le biais de l'abandon du foyer, la recherche d'un emploi décent et la vente de services sexuels.

Notre étude est centrée sur le Sud de la province de Buenos Aires pendant les années 1940, période de profondes transformations sociales et institutionnelles en Argentine. La crise économique de 1930 entraîne en effet une migration importante d'habitants des provinces et de la région agricole de La Pampa à la recherche d'un emploi dans cette métropole. Face au durcissement des formes de domination oligarchiques et à l'effondrement des marchés, l'État doit imaginer de nouvelles modalités d'intervention pour adapter une population au nouveau modèle de développement capitaliste afin de moderniser et d'industrialiser le pays ${ }^{6}$. Pour accompagner les politiques de soutien à l'industrie argentine encore balbutiante, se développe progressivement un ensemble de mesures sociales soutenues par des groupes réformistes qui vont des socialistes aux conservateurs ${ }^{7}$. Au fur et à mesure que les villes connaissent une transformation rapide due à l'exode rural, l'État affine ses outils d'intervention au sein des familles, en créant notamment la direction de la Maternité et de l'Enfance (1936) et la caisse de Maternité (1933), institutions qui se développeront avec la consolidation de l'État péroniste à partir de $1945^{\circ}$.

L'élargissement des droits sociaux coïncide avec des transformations normatives prenant appui d'abord sur des doctrines positivistes puis eugénistes. Ces changements reconfigurent les relations entre parents et enfants et pointent les comportements irréguliers d'enfants pauvres. La loi n ${ }^{\circ} 19.903$ du Patronage des Mineurs, promulguée en 1919, redéfinit les notions d'autorité parentale attribuées dans le Code civil argentin et donne à l'État le pouvoir de réguler la tutelle des enfants et adolescents considérés comme " mineurs délinquants » ou "matériellement et moralement abandonnés». Pour modifier le comportement de ces mineurs, la loi permet aux services judiciaires et à des acteurs privés (sociétés de bienfaisance, familles) de leur attribuer d'office une place rémunérée pour garantir leur subsistance. Ce changement légal est accompagné d'une série de réformes qui modifient fortement l'autorité paternelle, comme la loi qui en 1926 donne de plus amples pouvoirs aux mères célibataires ${ }^{9}$. En 1931, le Patronage National des Mineurs est réorganisé par décret et, en 1937, la loi $\mathrm{n}^{\circ} 4664$ crée les tribunaux pour mineurs ${ }^{10}$, même si dans l'aire géographique que nous avons étudiée, les jeunes restent encore à la charge de la justice correctionnelle et criminelle ou des juges de paix.

\author{
6. BarRancos Dora, \\ "Problematic modernity: \\ gender, sexuality, and repro- \\ duction in twentieth-century \\ Argentina", in Journal of \\ Women's History, no 18 (2), \\ 2006, p. 123-150. \\ 7. SURIANo Juan, La cues- \\ tión social en Argentina, \\ 1870-1943, Buenos Aires, \\ Agencia Nacional de \\ Promoción Científica y \\ Tecnológica, 2004. \\ 8. Biernat Carolina et RamaC- \\ cloтtı Karina, "Government \\ tutelage of mothers and \\ children in Argentina: admi- \\ nistrative structures, law, \\ and technical staff (1936- \\ 1955)", in História, Ciências, \\ Saúde-Manguinhos, \\ no 15 (2), 2008, p. 331-351. \\ 9. Cosse Isabel, Estigmas \\ de nacimiento. Peronismo \\ y orden familiar, Buenos \\ Aires, FCE, 2007; FreIDENRAIJ \\ Cecilia, «Intervenciones \\ policiales sobre la infancia \\ urbana. Ciudad de Buenos \\ Aires, 1885-1920 ", in \\ Revista Historia y Justicia, \\ no 1 (6), 2016, p. 7-19. \\ 10. Stagno, Una infancia..., \\ op. cit., p. 45.
}


11. Allemand, Sirvientes, criadas..., op. cit.

SCHEINKMAN Ludmila.,

"Pequeños huelguistas: participación de menores en los conflictos de la industria del dulce en Buenos Aires

en la primera década del siglo XX ", in Trashumante.

Revista Americana de Historia Social, $\mathrm{n}^{\circ} 1$ (8), 2016, p. 20-35.

12. Aversa María, «Las tramas sociales de la minoridad: infancias pobres y oficios "deshonestos" en la ciudad de Buenos Aires, fines del siglo XIX y principios del $X X$ », in Trashumante. Revista Americana de Historia Social, no 1 (8), 2016 p. 132-153; Cosse, Estigmas de..., op. cit.; GuY, Las mujeres..., op. cit.; STAGNo, Una infancia..., op. cit.

13. CalandRia Sol, "Maternidades en cuestión: modelos idílicos y prácticas de las madres en Argentina 1892-1936 ", in Trabajos y comunicaciones, $n^{\circ} 1$ (41),

2015, p. 15-32.

14. Biernat Carolina, RamacCIOTTI Karina, Crecer y multiplicarse. La política sanitaria materno infantil: Argentina 1900-1960, Buenos Aires, Biblos, 2013.

15. SuRIANo Juan, « ¿Cuál es hoy la historia de los trabajadores en la Argentina? », in Mundos do Trabalho, n० 1 (1), 2009, p. 27-50.
Les historiens des minorités considérées comme irrégulières en Argentine se sont intéressés aux enfances et aux jeunesses prolétaires urbaines ${ }^{11}$; ils ont analysé les activités de bienfaisance et de l'État dans l'arbitrage des pratiques sociales des minorités ${ }^{12}$; ils ont mis en avant les maternités ou les paternités considérées par la justice comme défectueuses ${ }^{13}$, et ils ont exploré le processus d'expansion des politiques sociales, renforçant le binôme mère-enfant ${ }^{14}$. Cette recherche a pour but de contribuer à enrichir ce champ sur deux plans : en analysant comment les mineurs ont agi pour négocier leur intimité et leur sexualité, mais aussi en élargissant cette étude à la vie des familles pauvres d'origine rurale dans des zones périphériques, généralement délaissées dans les recherches plutôt centrées sur les grosses villes ${ }^{15}$.

Les arguments défendus dans cet article sont les suivants : premièrement, les femmes cataloguées comme mineures ont dû faire face à des processus d'intervention publique et privée (familiale) qui les ont reléguées à une condition subalterne de genre et de classe. En tant que telles, elles sont devenues des médiations centrales de leur univers et de leurs expériences corporelles et sexuelles. Deuxièmement, les jeunes femmes ont développé des alternatives pour échapper aux normes sociales instituées notamment au sein de leurs familles. Parmi les tentatives déployées par ces dernières pour acquérir une relative autonomie figurent : la recherche d'un emploi dans une autre ville, la consolidation ou la rupture de l'union conjugale ou le concubinage et, à certaines occasions, la prostitution. Dans ce texte, nous observerons comment les jeunes filles ont développé des 
tactiques particulières et étudierons quelles sont les pratiques qui se sont révélées plus efficaces que d'autres.

À cette fin, nous analyserons les trajectoires de vie d'Elba, évoquée précédemment ${ }^{16}$, de Lucía (13 ans, de Ramón Santamarina ${ }^{17}$ ) et de Renée (16 ans, de Tres Arroyos-Mar del Plata $\left.{ }^{18}\right)$. Ces trois cas ont été choisis parmi un échantillon de 40 dossiers judiciaires du Département historique judiciaire du sud (DHJS) dans des affaires de proxénétisme, attentat à la pudeur, abandon du foyer, encouragement à la prostitution et détournement de mineurs. À partir de ces documents, nous avons réuni les témoignages de 68 femmes, de 140 hommes qui ont payé pour une relation sexuelle et de 100 parents et voisins. Ces dossiers ont été choisis car ils sont représentatifs des expériences traitées dans l'ensemble du corpus documentaire. Notre éventail d'analyse porte sur le département judiciaire sud de la province de Buenos Aires. Celui-ci inclut des petites localités (se consacrant à la production agricole), des villes moyennes (ayant des accès portuaires) et des grandes villes comme Mar del Plata.

Nous aborderons les récits de victimes, d'accusés, de plaignants, de témoins et de fonctionnaires ainsi que les paroles qui n'auraient pas pu être formulées autrement que dans le cadre d'une "normalité " perturbée ${ }^{19}$. Nous sommes conscients que ces documents présentent des scénarios polyphoniques dans lesquels cohabitent les voix de différents acteurs. Une lecture à rebrousse-poil doit être réalisée afin de percevoir les motivations des jeunes filles pour organiser leur recours face au système judiciaire ${ }^{20}$.

Ce travail de recherche comprend deux parties : la première, centrée sur Elba, étudie comment son statut légal de mineure, ses relations de genre et les rapports de classe ont un impact dans la construction de son intimité, analysant aussi les réactions de la mineure et de sa famille dans ce processus. Dans la deuxième, nous abordons la place de l'union conjugale et du concubinage comme médiation des interventions et des usages flexibles des normes qui ont joué un rôle dans ces expériences sexuelles (comme les régulations liées à l'emploi, la minorité, le mariage et la débauche).
16. DHJS, dossier $508: 22$ 1942.

17. DHJS, dossier 657:7, 1947 y 1948.

18. DHJS, dossier 657:7, 1947 y 1948.

19. FARGE Arlette, The allure of the archives, USA, Yale University Press, 2013.

20. Tenti María, «Los estudios culturales, la historiografía y los sectores subalternos ", in Trabajo y sociedad, n० 1 (18), 2012 , p. 317-329. 
21. Le travail des récoltes implique souvent le déplacement des hommes vers des fermes ou des propriétés éloignées de leur foyer pendant plusieurs jours du matin jusqu'au soir. Voir : VILLULla Juan, Las cosechas son ajenas: historia de los trabajadores rurales detrás del agronegocio, Buenos Aires, Cienflores, 2015.

22. Levı Giovanni, La herencia inmaterial: La historia de un exorcista piamontés del siglo XVII, Madrid, Editorial Nerea, 1990.

23. Ogden Johana, Between protection and punishment: children, sexual crimes and law in Buenos Aires, 18531912, Doctoral dissertation, Texas, University of Texas, 2015.

24. BarRancos Dora. Mujeres en la sociedad argentina: una historia en cinco siglos, Buenos Aires, Sudamericana, 2012.

\section{UN ÉTAT « À LA MAISON » : LES MÉDIATIONS ÉTATIQUES DANS LA VIE QUOTIDIENNE}

La plainte de María à propos du prétendu détournement de sa fille rend légitime l'intervention du tribunal criminel et correctionnel pour définir comment et où Elba a dû mener sa vie hors de sa famille. Les fonctionnaires établissent quels aspects de cette nouvelle configuration sont sains pour le développement idéal de la jeune fille. Sa relation avec Salvio, classée sans suite faute de preuves, a été pointée comme l'élément problématique central. Il est possible que la principale raison pour laquelle la mère a fait appel à la Police afin que sa fille soit ramenée là où elle avait été placée, était que c'était pour elle et sa famille un moyen d'obtenir de la nourriture quand son mari s'absentait pour les récoltes ${ }^{21}$. Mais cette réclamation n'aboutit pas puisque sa plainte conduit finalement à un placement en institution de la jeune fille.

En effet, quand les classes populaires ont recours à la justice, elles sont en général peu informées des tenants et aboutissants tant physiques et matériels que cela engendre. D'un côté, elles simplifient à l'extrême les mécanismes de cause à effet, de l'autre, elles utilisent consciemment les incohérences du système de règles et de sanctions. C'est pourquoi, il est possible de parler d'une rationalité sélective qui a tendance à expliquer les comportements individuels entre le comportement subjectivement désiré et celui socialement requis ${ }^{22}$. Dans cette partie, nous analyserons à travers la trajectoire d'Elba les frontières poreuses entre interventions publiques et interventions privées qui conduisent à définir les sexualités féminines considérées comme irrégulières.

\section{L'INCARNATION DE LA MINORITÉ :}

\section{QUE PEUT LE CORPS D'UNE JEUNE FILLE?}

La plainte pour détournement, qui présuppose une relation sexuelle douteuse entre une mineure et un adulte, entraîne un examen de « défloration $^{23}$ ». Cet examen est effectué par un médecin de la Police qui procède à un toucher vaginal pour vérifier l'état de l'hymen. Les organes génitaux féminins ont une charge symbolique forte et sont la métaphore de la bonne morale des femmes, mais sont par ailleurs le creuset de son intimité24. L'attouchement direct d'un homme est une pratique invasive qui violente l'identité de celle qui 
est examinée. Le médecin de la Police doit vérifier que la femme est vierge et, dans le cas contraire, établir depuis quand et pourquoi elle ne l'est plus (qu'il s'agisse d'une relation consentie ou non). Ensuite, le professionnel interroge sa patiente pour rédiger un rapport sur ses facultés morales, qui expliquent les causes du délit, et préconise éventuellement des actions judiciaires.

En répondant à cet examen, Elba signale qu'elle a perdu sa virginité avec le fils aîné de Giménez contre 2 \$. Pour le médecin, la lecture des cicatrices internes est la marque d'une "mineure abandonnée ", avec "peu de soins familiaux ». Ce verdict médical aura un rôle décisif dans la décision du juge qui ordonne de séparer la fille de ses parents ${ }^{25}$. Les discours médicaux et judiciaires coïncident pour reproduire un modèle normatif de maternité dans lequel la mère est rendue coupable de ne pas correspondre au rôle attendu - on peut ici y déceler l'empreinte de la loi du Patronage des Mineurs. On indique ainsi que la génitrice ne possède pas les facultés féminines considérées comme naturelles : le soin et l'éducation de ses enfants.

Comme nous l'avons décrit dans l'introduction, les interventions de ce type répondent au lent abandon du caractère libéral de l'État argentin pour un État davantage interventionniste. Lui-même a déployé de nouvelles politiques sociales quant au binôme mère-enfant dans la perspective de redéfinir ce lien ${ }^{26}$. Dans les zones rurales, l'État est dépendant de la capacité des tribunaux en tant qu'acteurs centraux dans la construction de l'ordre social et culturel ${ }^{27}$; raison pour laquelle on peut penser que l'action punitive sur les corps et les sexualités irrégulières s'inscrit dans un processus plus général.

La violence institutionnelle de l'examen du corps féminin est légitimée par les normes de genre et par l'âge d'Elba. Dans le discours public, la femme se construit ontologiquement en opposition à l'homme comme un objet passif dépositaire de la libido masculine $e^{28}$. Pour Nerio Rojas (1943), médecin reconnu, spécialisé en médecine légale et législateur national du parti socialiste-libéral de l'Union civique radicale $\left(\mathrm{UCR}^{29}\right.$ ), la certification de la « rupture totale de l'hymen » est la condition nécessaire pour déterminer la pureté d'une femme, et est révélatrice de sa bonne moralité.

Ces procédés considèrent les organes génitaux féminins comme pouvant potentiellement être altérés, contrairement au pénis masculin. Ils sont uniquement comparables à l'anus en tant que dépositaires potentiels de la féminité corruptrice du corps. Dans l'appareil reproducteur de l'homme, des marques

\author{
25. DHJS, dossier 508: 22, \\ 1942. \\ 26. Biernat Carolina et \\ Ramacciottı Karina, Crecer \\ y multiplicarse..., op. cit., \\ p. 12. \\ 27. Palacios Juan Manuel, \\ La Paz del trigo: cultura \\ legal y sociedad local en \\ le desarrollo agropecuario \\ pampeano, 1890-1945, \\ Buenos Aires, Edasha, 2004. \\ 28. Valobra Adriana, «El \\ Estado y las mujeres, \\ concepciones en clave femi- \\ nista ", in Estudios Sociales \\ del Estado, no 1 (2), 2015, \\ p. 33-57. \\ 29. Nerio Rojas (1890-1970) \\ est un médecin légiste et \\ un psychiatre argentin avec \\ une forte influence à la fois \\ dans son champ médical \\ comme auprès de l'État \\ argentin. II a été profes- \\ seur titulaire de la chaire \\ de médecine légale (1924 \\ à 1946) de l'université de \\ Buenos Aires, et en 1955, il \\ a occupé le poste de recteur \\ de cette institution. II a été \\ élu député national du parti \\ UCR pendant trois mandats \\ non consécutifs (1942-1943; \\ 1946-1950; 1960-1962). \\ Il a occupé des postes de \\ directeur pour d'importantes \\ publications spécialisées \\ et a été ambassadeur de \\ I'Unesco (1964-1966).
}


30. Revenin Régis,

" Conceptions et théories savantes de l'homosexualité masculine en France, de la monarchie de Juillet à la Première Guerre mondiale ", Revue d'Histoire des Sciences Humaines, $\mathrm{n}^{\circ} 17$, 2007, p. 23-45. [https://www. cairn.info/revue-histoire-dessciences-humaines-2007-2page-23.htm]

31. Simonetro Patricio, « Del consultorio a la cama. Discurso, cultura visual, erótica y sexología en la Argentina ", in Sexualidad, salud y sociedad, no 1 (22), 2016, p. 103-125.

32. Je fais référence au concept d'intersectionnalité pour expliquer les effets complexes, irréductibles et variés - économiques, politiques, culturels, psychologiques, subjectifs et expérimentaux - qui en dérivent quand de multiples axes de différenciation s'entrecroisent dans des contextes historiquement spécifiques, et constater que les différentes facettes de la vie sociale ne peuvent pas se séparer en formes discrètes et pures. Voir : Brah Avtar, Phoenix Ann, "Ain't I a woman? Revisiting intersectionality", in Journal of International Women's Studies, n’ 5 (3), 2004, p. $75-86$. d'un état naturel perverti ne peuvent pas être envisagées puisqu'il ne s'agit pas d'une entité passive potentiellement pénétrée. Ce n'est pas par hasard si la figure des uranistes $^{30}$ ou des homosexuels était conçue par la sexologie locale comme l'inversion de l'homme, alors considérée comme une femme, au moyen de la pénétration anale ${ }^{31}$. La figure de la mineure pure - celle qui n'a pas été pénétrée - est l'affirmation d'une représentation ontologique de l'être féminin qui, pour rester dans les normes respectables, ne peut abandonner cette condition qu'avec le mariage ou en atteignant l'âge adulte.

Le résultat de l'examen et la désignation d'Elba comme mineure, c'est-àdire une minorité légale fixée à moins de 21 ans, entérinent des prescriptions normatives qui alimentent la construction de sa condition subalterne. Comme nous l'avons expliqué précédemment, l'idée de minorité est mobilisée par l'élite politique et culturelle pour décrire des enfants et des jeunes ayant des comportements, une éducation et des sexualités considérés comme irréguliers. Être mineur fige ainsi un statut social de minorité légale face à l'autorité familiale, et ces mineurs sont alors susceptibles de subir les modes d'intervention spécifiques à travers lesquels, comme dans le cadre de l'ingérence médicale, se définissent les usages possibles de leur corps. Il est envisageable que la répétition de ces mécanismes, articulés avec des violences passées, invisibles dans nos documents, soient des éléments distinctifs de la genèse des concepts les considérant comme subalternes quant à leur sexualité, leur corps et leur intimité.

\section{À LA CROISÉE DE LA FAMILLE ET DU TRAVAIL :}

\section{LA CONSTRUCTION DE L'INTIMITÉ}

Être défini comme mineur est le symptôme institutionnel de l'intersection entre la subordination de l'État et celles du genre et de la classe ${ }^{32}$. Cela constitue une position secondaire face à leur famille et à l'État, qui aboutit à un statut légal conditionné par l'âge, et qui sert de médiateur dans les expériences sexuelles. Dans la vie des jeunes filles comme Elba convergent les limites légales, liées à leur âge et à leur genre, et les frontières de classe, qui délimitent un éventail de décisions possibles en raison même des difficultés à garantir leur subsistance.

Les registres des mineures suspectées de prostitution permettent de découvrir les conditions de vie des jeunes filles des secteurs populaires. Le fait d'être 
mineures les met dans une position de no man's land judiciaire : elles ont l'âge d'être employées mais sont sous la férule de leurs parents jusqu'à leurs 21 ans, et une fois en couple, sous celle de leur mari ou concubin. Depuis 1907, la loi 5291 a régulé à travers un régime spécial l'emploi des enfants âgés de moins de 10 ans et des femmes. En 1924, la loi 11317 sépare les règles entre le travail rural et urbain, augmente la limite de l'âge minimum légal de travail à 12 ans et interdit aux moins de 14 ans de travailler comme domestiques ${ }^{33}$.

De plus, être une femme pauvre dans la région Sud de la province de Buenos Aires accentue une existence précaire marquée par la pauvreté, les tâches ardues de la maternité et les possibilités réduites de trouver un emploi sur le marché du travail - ce qui réduit les marges d'autonomie. La primauté des cycles économiques ruraux et l'exportation de biens primaires marquent un marché du travail peu stable et mal rémunéré qui privilégie la force masculine ${ }^{34}$. La mécanisation de l'activité agricole et la fin de l'expansion territoriale horizontale pendant les années 1930 ont aggravé la pénurie d'emplois stables dans les fermes et les haciendas ${ }^{35}$. Entre 1930 et 1960, une masse considérable de travailleurs saisonniers se constitue. Les recensements d'expulsions permettent d'observer que face aux 15000 ouvriers permanents (qui se consacrent principalement à l'élevage) existent, dans la province, 9000 ouvriers saisonniers, employés pour la plupart dans la culture de légumes, le battage et le stockage du grain, travaillant de mars à juin et de novembre à janvier ${ }^{36}$. Quelques hommes et quelques femmes migrent alors de manière saisonnière à Mar del Plata, pendant les années 1940, la ville pouvant offrir un contrat dans la construction en hiver pour les hommes, d'août à janvier dans les usines de conserves et de poissons pour les hommes et les femmes, puis progressivement en été des emplois de saison $^{37}$.

Comme d'autres jeunes filles pauvres, Elba est placée comme domestique dans un foyer autre que le sien et ainsi contribue à faire vivre sa famille nombreuse ${ }^{38}$, de manière directe (apportant un revenu sous une forme ou une autre) ou indirecte (représentant une bouche en moins à nourrir ${ }^{39}$ ). Un tiers des 68 femmes répertoriées dans notre corpus d'archives sont employées comme domestiques lors de leur arrestation et presque la moitié d'entre elles avaient exercé cette profession avant leur majorité et avant l'âge de se marier ou de se mettre en concubinage (pour se consacrer aux tâches ménagères). Une grande partie des mères de ces jeunes filles ont déclaré qu'elles aussi avaient été placées
33. Allemandi, Sirvientes, criadas..., op. cit., p. 25.

34. AdAmovsky Ezequiel, Historia de las clases populares en la Argentina : desde 1880 hasta 2003, Buenos Aries, Sudamericana, 2013.

35. Balsa Juan, El desvanecimiento del mundo chacarero. Transformaciones sociales en la agricultura bonaerense 1937-1988, Bernal, Universidad Nacional de Quilmes, 2006.

36. VilluLla, Las cosechas..., op. cit., p. 55.

37. MolinARI Ines, «Obreras operarias y empleadas. El trabajo de las mujeres en Mar del Plata, entre los años 1940 y 1960 ", in Trabajos y comunicaciones, 1(34), 2008, p. 153-170 ; GaRAZZı Débora et Perez Inés, "Domestic and hotel Maids"-women's work, justice and inequality (Mar Del Plata, Argentina, 1956 to 1974)", in Cadernos pagu, no 1 (42), p. 313-340.

38. Une enquête de l'époque montre que dans les espaces urbains la majorité du salaire est utilisée pour l'achat de nourriture, ce qui constitue la moitié du salaire des pauvres. Bien que les aliments soient meilleur marché dans les villages, le prix monte pour la consommation d'autres biens de subsistance - comme les produits d'hygiène, les habits, les biens durables, etc.

39. Panter-Brick Carl et Smith Matthew, Abandoned children, Cambridge, Cambridge University Press, 2000. 
40. Données élaborées sur la base des trajectoires des femmes qui figurent dans les dossiers judiciaires recueillis du DHJD entre 1930 et 1960

41. Allemandi, Sirvientes, criadas..., op. cit., p. 15-65.

42. Milanich Nara, "Women, Children, and the Social Organization of Domestic Labor in Chile", in Hispanic American Historical Review, n० 91 (1), 2011, p. 29-62.

43. Barrancos, Mujeres en la..., op. cit., p. 178.

44. Losada Leandro, La alta sociedad en la Buenos Aires de la "Belle époque ", Buenos Aires, Siglo Veintiuno, Editora Iberoamericana, 2008.

45. DHJS, dossier 508: 22, 1942.

46. La question des arguments et de la légitimité du travail des mineurs lue à travers les causes judiciaires s'inspire de BLUM Anne, "Speaking of Work and Family: Reciprocity, Child Labor, and Social

Reproduction, Mexico City, 1920-1940", in Hispanic

American Historical Review, n० 91 (1), 2011, p. 63-95. quand elles étaient jeunes ${ }^{40}$. Faire entrer des mineures dans des maisons ne se pratique pas uniquement dans les zones rurales. Certaines études attestent qu'il existe à Buenos Aires, entre 1900 et 1940, une forte demande de filles et de garçons pour le service domestique. Ceci confirme qu'il s'agit d'une pratique habituelle chez les travailleurs appauvris, variable selon le marché local ${ }^{41}$. La reproduction intergénérationnelle de cette situation marque également les trajectoires familiales.

Entrer dans des maisons comme servantes est devenu constitutif de la classe subalterne des jeunes filles, que leur statut inférieur de genre et d'âge ${ }^{42}$ contribue à renforcer. Les jeunes femmes pauvres doivent passer par des situations d'inégalité face aux familles aux revenus plus importants, et cela a un impact sur leurs expériences sexuelles. Les services sexuels qu'Elba a fourni au fils du boulanger contre rétribution peuvent être lus à l'aune de traditions culturelles patriarcales comme un "droit de cuissage " dont le patron ou son fils peuvent jouir lors de la première nuit des jeunes femmes employées dans leur maison. Au-delà de ces prérogatives du patriarcat classique, pour les jeunes travailleuses, ces expériences sexuelles extra-matrimoniales sont également des pratiques courantes, même si elles entraînent parfois des conséquences ultérieures ${ }^{43}$. Par ailleurs, même si le fils du boulanger ne fait pas partie des élites locales, chez les familles argentines de rang élevé et peut-être au sein des classes moyennes, il est alors commun de penser que les jeunes domestiques garantissent l'accès à une première relation sexuelle sans risque de maladies vénériennes ${ }^{44}$. Elba remet l'argent à sa mère et à ses sœurs pour acheter de quoi manger, vraisemblablement parce que, lors des absences du père, les femmes organisent la vie de la maison et se débrouillent par elles-mêmes pour se nourrir. Élément qu'Elba n'hésite pas à mettre en avant dans sa déclaration au juge pour essayer d'éviter une mesure qui l'empêche de constituer son propre foyer ${ }^{45}$.

Pour les familles bénéficiaires, accepter de prendre une personne à gage était vécu comme une faveur octroyée au groupe domestique dont la jeune fille était issue, voire même comme une action qui les dote d'un statut dans la hiérarchie sociale locale. Elles s'appuient sur différents arguments pour légitimer cette situation ${ }^{46}$; Giménez explique l'emploi d'Elba comme une faveur faite à sa mère. De même que d'autres voisins, hommes et commerçants, convoqués pour donner leur avis sur le comportement moral de la mineure, il indique que la jeune fille travaillait chez lui afin de la préserver de tout comportement 
inadéquat ${ }^{47}$. Cet argument du service rendu, donné par le commerçant, explique que l'essentiel de la rémunération d'Elba soit versé à sa famille. Le fait qu'elle soit placée chez lui situe cette relation au croisement entre le travail, l'amitié, l'affectif et le domestique, et facilite le contournement d'un paiement officiel ${ }^{48}$. Maria apprend qu'Elba n'est plus chez le boulanger quand une de ses filles va y chercher «les biscuits et le pain » que la famille reçoit en échange du travail de la mineure ${ }^{49}$.

Elba abandonne la maison de Giménez pour vivre avec un homme plus âgé qu'elle, même si ce n'est pas une garantie de subsistance pour elle. Elle choisit une relation considérée illégitime qui finit par la conduire à un placement en

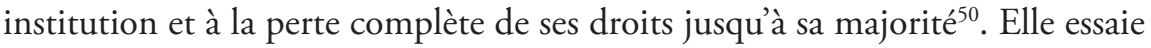
de contourner les limites et de créer sa propre intimité. Mais, il est rare que les jeunes filles obtiennent le résultat escompté.

En contestant le choix de leur fille parce qu'elle est mineure, la réaction familiale permet l'action publique. Comme nous l'avons identifié dans d'autres procédures judiciaires, les fonctionnaires essayent de reconstituer les rôles familiaux traditionnels. L'État a ainsi l'habitude de convoquer les parents pour désigner le «bon » foyer, celui qui convient aux soins des enfants. Bien que ce soit la mère d'Elba qui ait déposé la plainte, c'est la famille qu'elle représente qui se trouve finalement au banc des accusés au nom de représentations traditionnelles d'ordre, traversées par des notions de genre et de classe sociale. Pour cela, le tribunal fait appel à un groupe de voisins qui sont interrogés sur la conduite morale des parents. La plupart de ceux qui réprouvent le couple les définissent comme " extrêmement pauvres » et responsables d' " un manque d'attention » qui a pour résultante "quatre filles à la morale douteuse ${ }^{51}$ ". Les 63 témoins cités dans les quarante procès judiciaires enregistrés entre 1930 et 1960 sont tous des hommes, $69,78 \%$ sont mariés et la majorité d'entre eux $(60,3 \%)$ ont entre 35 et 65 ans. Ils sont aussi sans doute reconnus de par leur position sociale : plus de la moitié se déclarent propriétaires terriens ou commerçants (respectivement $20,63 \%$ et $31,74 \%{ }^{52}$ ). Pour le cas qui nous intéresse, quatre hommes mariés sont convoqués : un propriétaire terrien, un agriculteur, un commerçant et un coiffeur. Le juge décide de transférer Elba au quartier des mineures de la prison d'Olmos à La Plata : il confirme ainsi une intervention qui condamne les attitudes d'un milieu familial jugées indignes. La déchéance de la puissance paternelle, que la famille avait cédée provisoirement dans la

47. DHJD, dossier 508:22, 1942.

48. Perez Ines, Canevaro Santiago, "Languages of Affection and Rationality: Household Workers'

Strategies before the Tribunal of Domestic Work, Buenos Aires, 1956-2013", in International Labor and Working-Class History, no 1 (88), 2015, p. 130-149

49. DHJD, dossier 508:22, 1942.

50. DHJD, dossier 508:22, 1942.

51. DHJD, dossier 508:22, 1942.

52. Données élaborées sur la base des trajectoires des femmes qui figurent dans les dossiers judiciaires recueillis du DHJD entre 1930 et 1960 . 
53. SMith-ROSEnBERg Carol, Disorderly conduct: Visions of gender in Victorian America, Oxford, Oxford University Press, 1986. démarche de placement domestique, s'entend comme la marque étatique d'une ouverture qui prétend permettre à la jeune fille d'entamer une nouvelle vie.

Séparer les jeunes filles de leur foyer implique un processus de rupture avec les relations affectives qui construisent leur intimité. Le placement provoque le déracinement des mineures pauvres de leur groupe d'appartenance initial. Si les femmes de la bourgeoisie subissent, au moment de leur mariage, un processus de désaffiliation de leur monde originel pour conquérir une place dans une unité domestique autonome de celle de leurs parents ${ }^{53}$, les mineures doivent, elles, s'adapter à des foyers dont elles ne font pas partie, avec moins de ressources symboliques que celles qui se marient. Le placement suivant d'Elba l'éloigne de 371 kilomètres de son foyer de résidence, ce qui réduit à néant les possibilités de rester en contact avec ses parents ou ses sœurs.

En 1944, à 18 ans, Elba écrit au juge pour qu'il prenne en considération sa volonté de sortir de l'institution dans laquelle elle a été placée. Le fait d'écrire une lettre et de choisir des arguments précis pour convaincre le fonctionnaire démontre que ces jeunes filles placées, tout en concédant une partie de leurs libertés vis-à-vis de l'État, développent des capacités nouvelles d'agir. Bien que sa demande lui soit octroyée, pour bonne conduite, Elba ne peut sortir de l'institution qu'un an plus tard en raison d'une épidémie de scarlatine qui affecte le pavillon. En 1945, Elba est libre, sous la tutelle d'un fonctionnaire qui veille à son comportement jusqu'à ses 21 ans et l'aide à trouver un emploi comme domestique.

Le parcours d'Elba permet d'interroger l'interaction entre les actions quotidiennes et les interventions de l'État dans la construction de ce qui relève de l'ordre domestique. L'expérience de ces femmes permet de problématiser la question de l'opposition public/privé. Nous pourrions essayer d'évaluer dans quelle mesure l'intervention de l'État modèle la sphère privée. En ce qui concerne le cas d'Elba, influencée par sa condition de mineure, elle est au cœur de l'intersection entre genre, classe et âge, jouant ainsi un rôle dans son expérience corporelle intime et sexuelle.

\section{MARIS, CONCUBINS ET FEMMES SÉPARÉES}

En 1947, à Ramón Santamaria, une ville rurale, Lucía, âgée de 13 ans, est placée comme domestique par une association de bienfaisance, parce qu'orpheline de père et de mère. Des 68 femmes enregistrées dans notre corpus, $40 \%$ 
sont des orphelines qui sont entrées comme domestiques dans des maisons familiales ${ }^{54}$ par le biais d'accords divers, publics ou privés. En échange de ses services, la famille qui la reçoit lui fournit nourriture et logement. À l'âge de 14 ans, elle s'enfuit et se met en concubinage avec un maçon de dix ans son aîné, lequel selon ses déclarations la contraint en lui infligeant des châtiments à avoir des relations sexuelles avec des voisins contre de l'argent. Après leur séparation, Lucía s'installe dans un bar géré par une ancienne prostituée. Le lieu fonctionne comme une maison close en dehors de la loi $\mathrm{n}^{\circ} 12331$, qui depuis 1936 avait aboli le système qui réglementait le permis de proxénétisme en Argentine ${ }^{55}$. Opprimée par son compagnon, Lucía s'enfuit avec le fils de la propriétaire du bar, Juan, un journalier embauché au port de Necochea dans l'exportation de produits saisonniers ${ }^{56}$. Confortée par la plainte contre le maçon qui l'exploitait sexuellement, elle sollicite auprès de la justice qu'on lui permette de se marier, abandonnant ainsi son statut de mineure pour former un nouveau foyer en banlieue ${ }^{57}$.

En 1948, Lucía porte plainte au commissariat contre son mari qui l'oblige à faire commerce de son corps. Elle s'y présente avec Jeanne, une amie orpheline de 16 ans qui s'était enfuie quelques jours auparavant de chez son concubin qui lui infligeait des violences semblables. Lucía atteste que Juan la bat avec des barbelés, l'affame et la fait rester à genoux toute nue sur le sol en terre de leur maison en plein hiver pour l'obliger à se prostituer. Selon les notes du commissaire, elle supplie en pleurs qu'on la libère de cet enfer ${ }^{58}$.

Avoir un concubin ou se marier pour les jeunes filles pauvres représente une voie possible pour créer un espace intime autre que celui de leurs parents ou de celui de la maison où elles sont placées. Entrer dans ces relations implique un changement quant à la place qu'elles occupent au sein du foyer. Comme toute pratique intergénérationnelle, être employée comme domestique ou s'unir à un homme fait partie des possibilités offertes à ces jeunes filles. Dans cette partie, nous explorerons les conditions dans lesquelles les mineures pauvres suspectées de prostitution ont négocié leurs expériences sexuelles.

54. Données élaborées sur la base des trajectoires des femmes qui figurent dans les dossiers judiciaires recueillis du DHJD entre 1930 et 1960 .

55. En 1936, la loi de prophylaxie sociale abolit la prostitution réglementée, c'est-à-dire, répertoriée dans les registres locaux. Voulant diminuer la propagation des maladies vénériennes, l'État déploie un ensemble de politiques parmi lesquelles se trouve cette mesure. En 1944, la loi est modifiée par décret du gouvernement militaire donnant la possibilité d'installer des maisons closes près des casernes. En 1955, la tentative de Perón de revenir au règlementarisme est anéanti par le nouveau coup militaire.

56. La ville portuaire de Necochea compte alors 17708 habitants.

57. DHJS, dossier 657:7, 1947.

58. DHJS, dossier 657:7, 1948. 
59. Escudero Pedro, La alimentación de la familia en Buenos Aires, Buenos Aires, Instituto Nacional de Nutrición, 1939.

60. HJS, dossier 657:7 1947.

61. Barrancos, Mujeres en la.., op. cit., p. 40-50.

62. DHJS, dossier 657:7

\section{NÉGOCIER L'INTIMITÉ : FAIRE APPEL À LA JUSTICE}

\section{ET CHERCHER DE L'ARGENT}

Les faibles revenus des hommes pour subvenir aux besoins de leur couple et de leur famille poussent les femmes à chercher ailleurs des ressources : travaux de blanchisserie, service domestique, emploi informel ou prostitution. Contrairement aux données de l'Institut national de nutrition qui indiquent que $96 \%$ des époux ont à leur charge leur épouse ${ }^{59}$, dans les régions où les revenus dépendent des récoltes, il est possible que les femmes soient obligées de chercher d'autres formes de rémunération pour garantir leur subsistance pendant la période de chômage de leur mari ou lors des longues absences des journaliers ruraux ou des dockers.

Ces femmes ont recours à différentes stratégies pour négocier leurs faveurs. Lucía le fait à plusieurs reprises. Tout d'abord, elle abandonne le bar où elle travaille en se mettant en concubinage avec un maçon; ensuite à travers son mariage avec Juan, elle se débarrasse de sa condition de mineure; enfin, en dernier recours, quand le juge l'interroge sur ses futurs projets, elle lui explique vouloir «s'installer" avec un de ses clients, un maçon de 23 ans $s^{60}$.

Le mariage est une institution privilégiée pour permettre l'action de l'État. L'union repose sur des normes juridiques, sociales et culturelles qui fixent une inégalité des époux dans l'espace domestique. L'État encourage un modèle de relation matrimoniale dans laquelle, jusque dans les années 1960, les femmes sont soumises à la tutelle masculine et ne peuvent pas accéder librement à la disposition de leurs biens ${ }^{61}$. Quand Juan, le mari de Lucía, explique à la justice pourquoi la plaignante a un œil « au beurre noir ", il affirme qu'elle s'est absentée de la maison sans lui avoir préparé à manger alors qu'il rentrait du travail. Le défenseur des pauvres - un avocat commis d'office par l'État pour Juan - estime justifié cet acte tout à l'instar de certains témoins qui signalent la moralité douteuse de Lucía. Le fait que la jeune femme ne donne pas d'explications sur l'endroit où elle se trouve légitime aux yeux des congénères de Juan la violence de ce dernier. En théorie, nous pourrions douter des déclarations de Juan, puisqu'il croit à juste titre que celles-ci seront validées par d'autres interlocuteurs, et nous pourrions ainsi nous demander si ce passage à tabac, comme tous les autres, n'est pas la résolution habituelle d'un conflit récurrent à propos des gains de Lucía ${ }^{62}$. 
L'espace domestique se trouve formalisé et renforcé par le mariage même si certains choisissent aussi le concubinage pour parvenir aux mêmes résultats. La vie commune entre hommes et femmes est tissée autour d'inégalités où le genre et l'âge réduisent les femmes à une condition auxiliaire. Juana, la jeune fille qui accompagne Lucía à la Police, précise que sa situation est similaire. Orpheline, elle a été placée comme domestique dès l'âge de 14 ans dans une maison, où elle a été victime de mauvais traitements. C'est ainsi, qu'après s'être enfuie, elle a eu des relations sexuelles avec différents hommes en échange du gîte et du couvert. Tout comme Lucía, elle déclare qu'elle s'est mise en concubinage avec un homme plus âgé, qui travaille comme employé municipal, et qui lui inflige différentes tortures pour la forcer à avoir des relations sexuelles avec d'autres hommes contre de l'argent ${ }^{63}$.

La violence maritale, renforcée par la relation de proxénétisme, est un mécanisme extrême de domination masculine au sein de l'espace domestique. Pour éviter les violences, Lucía doit donner la totalité de l'argent qu'elle gagne. Son tarif est de $7 \$$ pour des rapports sexuels chez elle ou de $50 \$$ pour passer la nuit avec un client. Sans horaires, les rencontres peuvent avoir lieu le matin ou le soir et ne durent pas plus de trente minutes, ce qui lui permet de mieux gagner sa vie qu'avec la rémunération précaire perçue comme domestique (40\$).

Le concept masculin d'appropriation de l'argent de sa femme en tant qu'affirmation de sa position masculine à l'intérieur de la cellule familiale tranche avec certaines images de l'époque. Dans les centres urbains, au milieu des années 1940 , les publicités s'adressent aux femmes des "classes moyennes ${ }^{64}$ " comme des agents de consommation dans leur rôle de femmes au foyer. Les femmes pauvres des zones rurales ne sont pas concernées par cette stratégie publicitaire. Cependant, l'absence des hommes du foyer liée aux cycles de travail, comme Juan qui passe une grande partie de la journée à charger dans le port ou qui part plusieurs jours travailler dans une ferme, laisse aux femmes le contrôle et la responsabilité du foyer. Cela encourage aussi les femmes à trouver dans la prostitution des ressources complémentaires à leur travail précaire, leur laissant parfois du temps libre et une certaine marge d'autonomie. Ce qui ne manquait de générer des conflits au sein du couple pour l'affirmation des rôles respectifs, la distribution des tâches et les attributions des hommes, femmes et enfants au sein du foyer.
63. DHJS, dossier 657:7, 1947.

64. PITE Rebekah, Creating a Common Table in Twentiethcentury Argentina: Dona Petrona, Women, and Food, USA, UNC Press, 2013. 
65. DHJS, dossier 657:7

66. DHJS, C525:28, 1940

67. DHJS, C525:28, 1940
Dans le cas de Lucía, pouvoir s'adresser aux institutions publiques en ayant une connaissance réduite des éventuelles retombées, démontre un usage flexible de la loi. Elle ne connaît pas exactement les règles du droit mais son expérience antérieure des procédures judiciaires lui apporte peut-être une aptitude intuitive à identifier la meilleure marche à suivre, aussi bien en se mariant (quand elle sollicite Juan pour l'épouser) qu'en se séparant (quand elle demande qu'il soit jugé pour proxénétisme).

Les deux situations rendent complexes les perceptions contradictoires par ces femmes du mariage comme élément central de leurs expériences et de leurs pratiques en lien avec les usages et interventions propres à l'État. Quand le procureur lui demande pourquoi elle s'est mariée avec Juan, elle explique que " comme elle était seule et sans protection, elle croyait qu'elle serait mieux traitée $^{65}$ ". Dans leurs déclarations judiciaires et face à leurs pairs, les femmes mettent en avant leurs qualités féminines en envisageant le mariage comme un moyen de collaborer avec leur famille. Dans ces comptes rendus, elles réaffirment les valeurs structurantes de la féminité, telles que la vertu, l'honneur et l'innocence.

En 1940, à Tres Arroyos, une communauté rurale au Sud de la province de Buenos Aires, Renée, 14 ans, épouse un maçon, de sept ans son aîné, avec la permission de sa mère Ramona, une veuve de 50 ans. Le mariage dure 45 jours parce que, selon les dires de la mère, elle ne supporte plus les sévices de son époux. Après avoir été un temps séparée, elle se met en concubinage avec Matías, un boulanger avec qui elle déménage à Mar del Plata pour chercher du travail. Un an après leur installation dans la ville, elle porte plainte contre son concubin pour proxénétisme ${ }^{66}$.

Dans une lettre que Renée envoie à sa mère, elle souligne que cette union soulage les dépenses domestiques de Ramona pour l'entretien de ses trois filles mineures. Quand la mère est interrogée par le juge, elle affirme « avoir eu l'espoir que cela lui apporterait une meilleure vie ». On peut penser que l'ensemble de ces échanges, le fait d'écrire, de témoigner pour ces femmes leur permet d'organiser leurs expériences domestiques et donne un sens cohérent à leurs décisions ${ }^{67}$.

Il y a plusieurs manières d'interpréter ces récits. Premièrement, ces affirmations compliquent les prescriptions issues des élites, à travers lesquelles nous considérons souvent que les agents publics construisent les objets et les sujets 
de leur politique. Même si l'État juge avec ses paramètres et ses frontières de ce qui est socialement admissible, contrastant généralement avec les réalités des travailleurs pauvres, il est possible que ces derniers transgressant les frontières de classe intègrent les normes sociales qui leur sont imposées. Les témoignages judiciaires des femmes pauvres montrent que, peut-être, dans leur quête de justice, elles adhèrent et placent un certain espoir dans les représentations dominantes du mariage.

Deuxièmement, pour nuancer cette première affirmation, nous pouvons préciser que, malgré les multiples interventions de l'État et les potentielles coïncidences sémantiques avec certaines notions dominantes autour de la nuptialité, les femmes ont négocié les conditions de leur intimité. Les expériences réitérées de Lucía avec la justice lui ont laissé espérer que les poursuites contre Juan seraient une aide efficace contre la violence de son mari et pour récupérer son argent. De son côté, Renée a été capable de passer du statut de femme mariée à celui de concubine avec un autre homme, sans quoi elle ne serait pas parvenue jusqu’à Mar del Plata.

Troisièmement, le salut que les femmes croient trouver dans leurs unions est variable. Le fait qu'elles soient femmes ne les empêche pas d'avoir des pratiques alternatives face aux multiples inégalités subies dans leur vie domestique. Arrivée à Mar del Plata, avant de porter plainte contre son mari pour proxénétisme, Renée et son concubin partagent leur logement avec un autre couple. C'est là qu'elle devient l'amie d'Elvira, avec laquelle elle se balade dans la ville à la recherche de clients, pendant que les hommes travaillent (l'un comme boulanger et l'autre comme maçon).

Lors de ces promenades, les femmes cherchent plus que de l'argent pour leurs économies domestiques, elles s'adonnent au flirt. L'idée moderne d'amour romantique a cherché à bien distinguer l'économie des sentiments ${ }^{68}$. L'anthropologie et la sociologie soutiennent l'idée que les prostituées établissent des limites pour classer leurs actes en séparant les sphères de l'argent et de la sexualité ${ }^{69}$. Avec certains clients, Elvira et Renée développent de complexes rituels qui incluent des échanges matériels et symboliques. Ainsi la rencontre avec des employés du cinéma leur permet de se voir offrir des bas d'une mercerie, de se faire inviter au cinéma et donner des petits gâteaux en guise de séduction.
68. GIDDENS Antony, La transformation de l'intimité : sexualité, amour et érotisme dans les sociétés modernes, Paris, Hachette, 2006.

69. Morcillo Santiago, «Mujeres invisibles: Políticas del ocultamiento entre mujeres que hacen comercio sexual ", in Trabajo y sociedad, no 1 (29), 2017, p. 41-60. 
70. L'asado (« grillade »), très répandu en Argentine, rassemble des convives pour déguster de la viande grillée (note de la traductrice)

71. Selon les données extraites lors de la grève des pêcheurs, elle gagnait $2,70 \$$ de par sa condition féminine, soit $2 \$$ de moins que les hommes. Voir :

Ruocco Laura, "De las obreras de la conserva a las fileteras. Cambios y continuidades del trabajo femenino en la industria del pescado. Mar del Plata 1942-1975 », in Revista de Estudios Marítimos y Sociales, $n^{\circ} 1$ (1), p. 127-130.

72. DHJS, C525:28, 1940.
La notion de "cadeau " dans leurs témoignages peut faire l'objet de différentes interprétations. Tout d'abord, comme un mot utilisé par crainte d'être qualifiées par l'État de prostituées. Même si aucune d'entre elles n'enfreint la loi, puisque celle-ci ne pénalise pas la prostitution en tant que telle. Ensuite, ces objets peuvent s'apparenter à un jeu de séduction, conférant à ces clients une place à part, plus romantique, soulignant dès lors les frontières complexes entre intimité et économie.

Le soir, ils les invitaient à dîner et passaient la nuit avec les jeunes filles moyennant 10 \$ chacune. Une somme attrayante sachant que ces femmes ont un loyer mensuel de 20 \$. Le badinage permet également à Renée d'accompagner l'un de ses clients à un $a s a d o^{70}$ ou encore de faire un tour de voiture en ville avec lui. Par la fréquentation de ces hommes, les femmes sont capables d'obtenir des gratifications monétaires et affectives. Quelques temps après, Renée entre comme ouvrière dans une usine de conserves de sardines. Avec ses maigres revenus et les gains obtenus grâce aux services sexuels qu'elle offre à quelques jeunes hommes pendant son temps libre, elle prend son indépendance, se séparant de Matías, pour s'installer dans une pension ${ }^{71}$. Par ailleurs, elle fait appel à la justice pour réduire les capacités d'action de son concubin ${ }^{72}$.

En somme, l'union et la séparation avec les hommes sont également des voies alternatives pour former l'espace domestique et privé. Tandis que l'État favorise l'asymétrie domestique par une répartition inégale des droits entre femmes et hommes, les femmes, dans certaines situations extrêmes complètent leurs revenus occasionnels soit en trouvant un appoint économique (Renée), soit en initiant un nouveau couple (Lucía). L'intervention publique leur permet alors d'obtenir une ouverture vers de nouvelles formes d'existence.

\section{Conclusion}

Dans cet article, nous avons étudié dans quelles conditions et au moyen de quelles pratiques les jeunes femmes comparaissant devant les tribunaux pour avoir vendu leur corps ont négocié leur intimité. Dans ce sens, nous avons constaté que la place secondaire de ces filles face à leur famille, employeurs ou conjoints, a permis à ces jeunes filles d'avoir conscience de leurs corps. Mais, faisons remarquer que loin de se soumettre à une condition de victimes passives, elles ont élaboré différentes stratégies pour modifier leur état, même si souvent elles n'obtiennent pas le résultat escompté. 
De plus, bien que pendant les années 1940 le pouvoir des institutions charitables et philanthropiques s'est réduit au profit des nouvelles politiques sociales materno-infantiles ${ }^{73}$, la continuité des pratiques menées par l'État (institutionnalisation) au moyen d'arrangements intrafamiliaux (placement) questionne les interprétations classiques et met l'accent sur la porosité entre la dimension publique et privée dans la construction des expériences sexuelles féminines.

À cet égard, malgré des résultats qui leur sont défavorables, les expériences des jeunes filles ne sont pas totalement modifiées par les normes. La constitution d'un foyer en fonction des relations conjugales ou de concubinage montre les limites des projets d'une vie hors de leur famille d'origine (si elles en ont) et hors d'un soutien financier masculin. Ce phénomène combiné aux restrictions sociales pour intégrer un monde du travail instable, constitue un ensemble de pressions contre lesquelles ces femmes se sont battues pour garantir leur subsistance. Entre l'intervention publique et les usages flexibles de la loi, apparaissent des situations, dans lesquelles elles ont assumé des risques en définissant d'autres usages de leur corps. Parmi ceux-ci, elles ont essayé d'obtenir des emplois formels, de se marier ou de se séparer et parfois de faire commerce de leurs corps.

Le complexe équilibre entre les marges d'action, les usages de la justice et les procédures d'intervention de l'État sont autant d'éléments qui éclairent le passé et permettent de nuancer l'idée d'un contrôle normatif institutionnel sur les actions quotidiennes des hommes et des femmes. Les diverses dépossessions que nous avons décrites dans cet article ne contredisent pas les capacités d'agir de ces femmes. Les situations variables dans lesquelles elles agissent, en général sans conscience des risques ou des conséquences, reflètent leur passage d'une forme domestique à une autre : de la domesticité au concubinage, de la plainte policière au travail mal payé ou à une institution pour mineurs.

Toutes cherchent, dans les interstices, à survivre dans des mondes marqués par des expériences précaires. Les actes quotidiens, les petites décisions qui placent Elba, Lucía ou Renée dans une situation de subordination domestique ou qui leur permettent de créer une quelconque relation affective, ne naissent pas d'un froid calcul stratégique. Elles relèvent de pratiques discontinues à partir desquelles elles participent d'une réélaboration de leur intimité et de leur condition domestique. Même si ce n'est pas toujours avec les résultats escomptés, à l'instar du sort d'Elba qui doit passer toute sa jeunesse enfermée en institution,

\author{
73. GuY, Las mujeres..., \\ op. cit.
}


les frontières poreuses entre le public et le privé permettent d'ouvrir des brèches pour l'intervention, la négociation et l'action, dans lesquelles se dessine le territoire complexe des domesticités pauvres. 